\title{
Design of a 3-Stage ADR for the Soft X-Ray Spectrometer Instrument on the Astro-H Mission
}

\author{
Peter J. Shirron*, Mark O. Kimball, Donald C. Wegel, Edgar R. Canavan, Michael J. DiPirro \\ NASA/Goddard Space Flight Center, 8800 Greenbelt Rd., Greenbelt, MD, USA 20771;
}

\begin{abstract}
The Japanese Astro-H mission will include the Soft X-ray Spectrometer (SXS) instrument, whose 36-pixel detector array of ultra-sensitive x-ray microcalorimeters requires cooling to $50 \mathrm{mK}$. This will be accomplished using a 3-stage adiabatic demagnetization refrigerator (ADR). The design is dictated by the need to operate with full redundancy with both a superfluid helium dewar at $1.3 \mathrm{~K}$ or below, and with a $4.5 \mathrm{~K}$ Joule-Thomson (JT) cooler. The ADR is configured as a 2-stage unit that is located in a well in the helium tank, and a third stage that is mounted to the top of the helium tank. The third stage is directly connected through two heat switches to the JT cooler and the helium tank, and manages heat flow between the two. When liquid helium is present, the 2-stage ADR operates in a single-shot manner using the superfluid helium as a heat sink. The third stage may be used independently to reduce the time-average heat load on the liquid to extend its lifetime. When the liquid is depleted, the 2nd and 3rd stages operate as a continuous ADR to maintain the helium tank at as low a temperature as possible - expected to be $1.2 \mathrm{~K}$ - and the 1st stage cools from that temperature as a single-stage, single-shot ADR. The ADR's design and operating modes are discussed, along with test results of the prototype 3 -stage ADR.
\end{abstract}

Keywords: adiabatic demagnetization refrigerator, space cryogenics, $x$-ray microcalorimeters

*Peter.Shirron@nasa.gov; phone 1301 286-7327; fax 1301 286-0389

\section{INTRODUCTION}

The SXS instrument on Astro- $\mathrm{H}^{1}$ will perform imaging spectroscopy in the soft $\mathrm{x}$-ray band using a $6 \mathrm{x} 6$ array of silicon microcalorimeters. By cooling the detectors to very low temperature $(50 \mathrm{mK})$, the energy of individual x-rays can be determined with a resolution of better than $4 \mathrm{eV}$ for energies up to $6 \mathrm{keV}$. The array will be cooled with a 3-stage ADR whose design and configuration were dictated by the need to operate redundantly with a hybrid superfluid helium dewar/Joule-Thomson cryocooler system ${ }^{2}$. That is, the ADR is required to meet its performance requirements using either the liquid helium as a heat sink, or the JT cooler after the liquid is depleted. This requirement applies even in the case of a JT cooler failure, as long as liquid helium is still present. The hybrid concept was developed as a result of the failure of the Astro-E2 helium dewar, and includes two fundamentally different coolers to minimize the possibility that a single failure mechanism could compromise both systems.

In order for the ADR to operate with two significantly different heat sink temperatures $(<1.3 \mathrm{~K}$ for the liquid helium and $4.5 \mathrm{~K}$ for the JT), it is composed of two separate assemblies: a 2-stage ADR that interfaces directly with the helium tank, and a $3^{\text {rd }}$ stage that interfaces to both the tank and the JT cooler. When liquid helium is present, as is expected for the nominal 3-year mission, only the 2-stage ADR needs to be operated to cool the detectors. Using the $\sim 1.2 \mathrm{~K}$ liquid as a heat sink, the 2-stage ADR's hold time is estimated to be $>100$ hours, much longer than the requirement of 24 hours. During this time, the $3^{\text {rd }}$ stage may be operated to transfer heat from the helium tank to the JT to extend the lifetime of the liquid. This will be particularly valuable should any of the Stirling shield coolers fail, resulting in higher parasitic heat loads on the cold stages of the dewar.

When the liquid is depleted - either normally or prematurely - the ADR's operation is fundamentally different. The $3^{\text {rd }}$ stage will be used to cool the helium tank, cycling back and forth to offload heat from the tank and transfer it to the JT. Based on nominal tank loads, the temperature can be maintained at an average value of $1.2 \mathrm{~K}$. The $2^{\text {nd }}$ stage will be used to stabilize the base of the detector assembly at a constant temperature, and the $1^{\text {st }}$ stage will operate as a single-stage ADR to cool the detectors. The use of the $2^{\text {nd }}$ stage only for stabilizing the detector housing temperature is necessary because detector gain shifts may occur if the temperature fluctuates by more than $1 \mathrm{mK}$ over a 10 minute period, a 
sensitivity that was seen on Astro-E2. Without this control, the periodic heat lift of the $3^{\text {rd }}$ stage $(1-2 \mathrm{~J}$ of heat every $\sim 40$ minutes) will cause short-term fluctuations of $\sim 50 \mathrm{mK}$. Although operating in this manner reduces the hold time of the $1^{\text {st }}$ stage considerably, to about 30 hours, it is the only way to meet all of the requirements levied on the ADR.

All operating modes of the ADR have been extensively modeled in order to estimate hold and recycle times, and to calculate parameters needed for transient analyses of the cryogenic system: current and temperature profiles, instantaneous heat loads from the ADR to the helium tank and JT cooler, etc. This paper describes the design and operation of the 3-stage Astro-H ADR, and reports on measured and predicted performance in the context of the SXS cryogenic system.

\section{CRYOGENIC SYSTEM CONFIGURATION AND REQUIREMENTS}

The SXS cryogenic system ${ }^{2}$ is shown schematically in figure 1 . The components enclosed in the dotted boxes will be supplied by NASA/GSFC: the ADR, the detector assembly and an interface plate that provides the mechanical and thermal interface to the helium tank. Collectively these components are referred to as the Calorimeter Spectrometer Insert (CSI). All other components of the cryogenic system are supplied by JAXA/ISAS: the helium dewar, including shields, and the 2-stage Stirling (2ST) and ${ }^{4} \mathrm{He}$ JT cryocoolers. To simplify the US/Japanese interface, the CSI is a single, integrated module that will be installed in a well at the top of the helium tank. As shown in Figure 2, the $1^{\text {st }}$ and $2^{\text {nd }}$ ADR stages are located in the well, while the detector assembly and $3^{\text {rd }}$ ADR stage are mounted to the top of the $\mathrm{CSI} /$ dewar interface plate. This allows the $3^{\text {rd }}$ stage to be connected to the JT cooler cold head - at the bottom of the helium tank - through a semi-rigid thermal strap. The helium tank and CSI are surrounded by an aluminum "JT" shield at approximately $4.5 \mathrm{~K}$, and additional shields cooled by vented helium vapor and by Stirling cryocoolers to minimize parasitic loads on the JT cooler. The ADR's magnets are powered by external supplies through leads consisting of warm resistive wires that transition to YBCO leads at the $20 \mathrm{~K}$ shield.

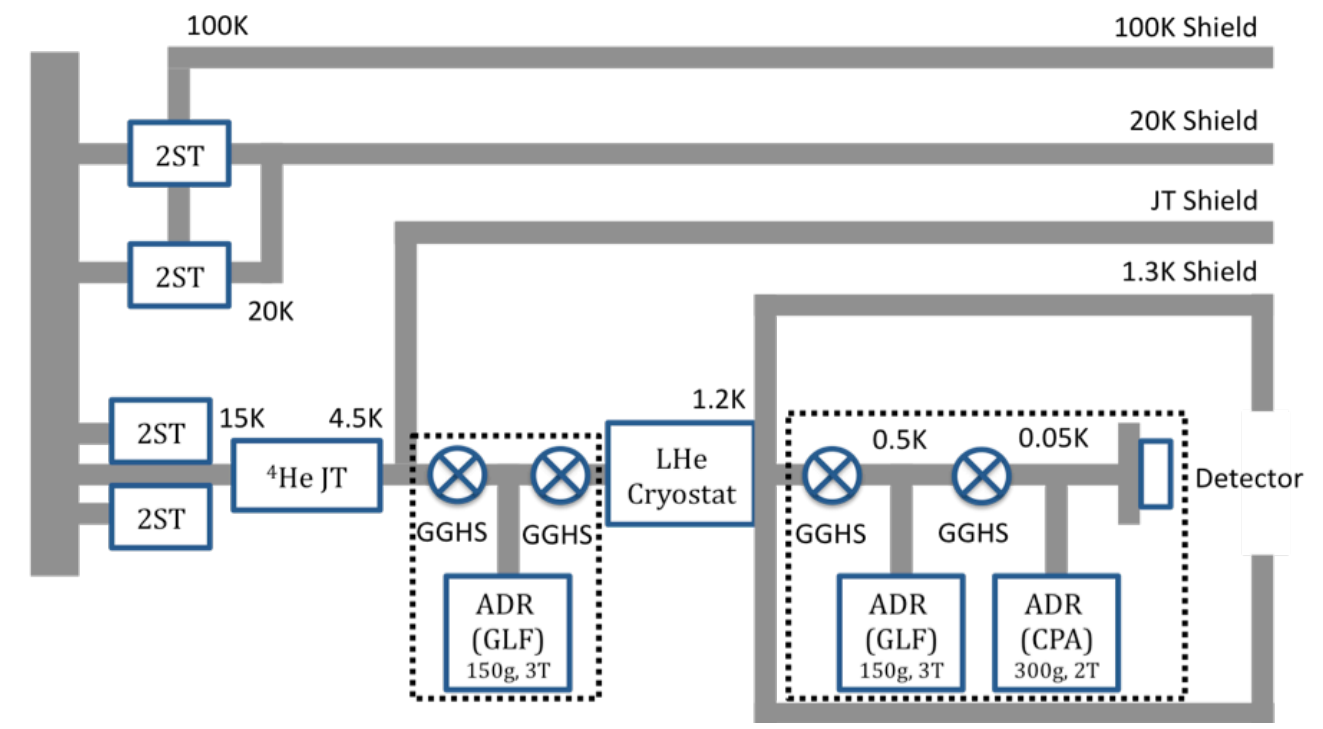

Figure 1. Schematic of the SXS cryogenic system. Redundant 2-stage Stirling (2ST) coolers are used to cool the dewar shields and as a pre-cooler for the JT cooler. The dashed boxes identify components that will be supplied by NASA/GSFC.

Selected requirements and parameters for the SXS instrument and ADR are listed in Table 1. The lifetime requirement of 3 years, when applied to the 30 -liter helium dewar, translates to a time-average heat load of less than $1.0 \mathrm{~mW}$. For the goal of 5 years, the total load must be less than $0.6 \mathrm{~mW}$. The thermal budget apportions this among parasitic loads from the dewar structure and harnessing, and a time-averaged load from the ADR. Combined with the dissipated heat by the detectors at $50 \mathrm{mK}$, the allotment of $0.21 \mathrm{~mW}$ is used to derive top-level performance parameters of the ADR, which are then translated into a detailed design. (These are summarized in Table 2.) Most importantly, the high efficiency implied 

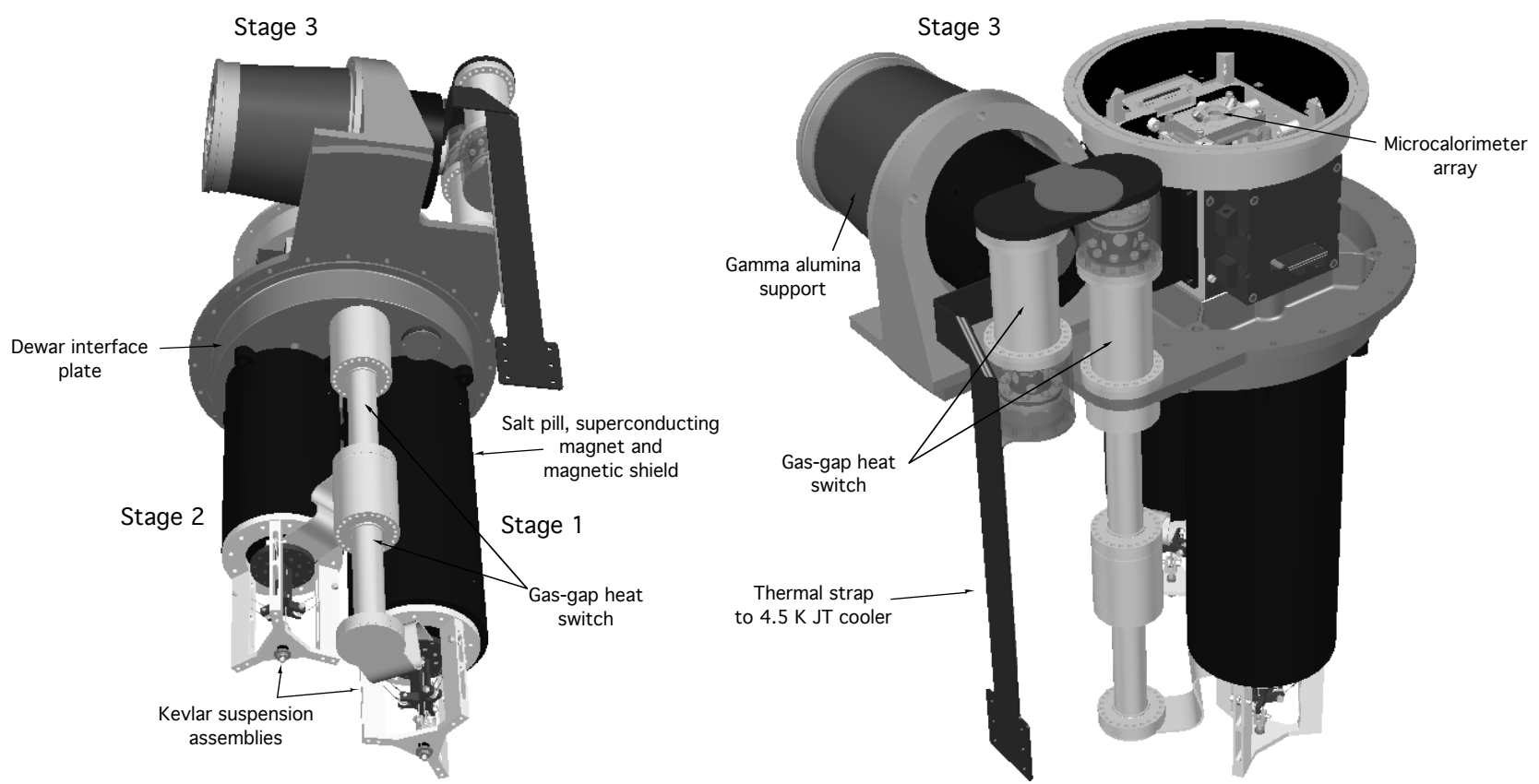

Figure 2. Bottom and top views of the CSI, highlighting the 3-stage ADR and SXS detector assembly.

in the budget biases the design toward 2 stages to bridge the gap from $1.2 \mathrm{~K}$ to $50 \mathrm{mK}$. It also turns out necessary to have two stages in order to meet the detector housing stability requirement when the JT is used as the heat sink. When the dewar contains liquid helium, the liquid's heat capacity provides the required stability. However, when the JT is used, the operation of the $3^{\text {rd }}$ stage involves periods when the stage is absorbing heat from the helium tank - and there is a net flow of heat out of the tank - and periods where the tank is absorbing parasitic loads - and there is a net flow of heat into the tank. This would give rise to a sawtooth temperature pattern of the cryogen tank and CSI, including, most importantly, the detector assembly, with a 20 minute period and $50 \mathrm{mK}$ amplitude. Only by having two stages connected between the tank and detector array is it possible to dedicate one stage to damping out these fluctuations and still have a stage available to cool the detectors. The operation is described in detail below.

Table 1. SXS instrument requirements.

\begin{tabular}{|ll|}
\hline Mission duration & 3 years, with a goal of 5 years \\
Detector resolution/band & $7 \mathrm{eV}$ for energies up to $6 \mathrm{keV}$ \\
Detector operating temperature, $\mathrm{T}_{\mathrm{op}}$ & $50 \mathrm{mK}$ \\
Detector temperature stability & $2.5 \mu \mathrm{K} \mathrm{rms}$ \\
ADR hold time at $\mathrm{T}_{\mathrm{op}}$ & $>24$ hours \\
ADR recycle time & 1 hour (2 hours for JT-based operation) \\
Detector heat load at $\mathrm{T}_{\mathrm{op}}$ & $<0.5 \mu \mathrm{W}$ (with a housing temperature of $1.3 \mathrm{~K}$ ) \\
Detector housing temperature stability & $1 \mathrm{mK}$ over 10 minutes (at $\sim 1.3 \mathrm{~K})$ \\
Time average heat load to liquid helium & $0.21 \mathrm{~mW}$ \\
Peak heat load to JT cooler & $20 \mathrm{~mW}$ (6 mW in case of failed shield cooler) \\
Redundancy & Full redundancy in operation with superfluid helium \\
& or the $4.5 \mathrm{~K}$ JT cooler \\
Fault tolerance & Full instrument performance if the superfluid helium \\
& dewar OR the JT cooler fails \\
\hline
\end{tabular}




\section{ADR DESIGN}

The ADR design builds on as much heritage from prior programs as possible (Astro- $\mathrm{E}^{3}$ and Astro-E2/Suzaku ${ }^{4}$, the $\mathrm{XQC}$ rocket program ${ }^{5}$, and the High-resolution Airborne Wide-band Camera (HAWC) ${ }^{6}$ and Submillimeter and Far InfraRed Experiment (SAFIRE) ${ }^{7}$ instruments on the Stratospheric Observatory For Infrared Astronomy (SOFIA)), but makes a significant departure from these designs in its use of multiple stages to achieve higher performance, efficiency, and the ability to operate with multiple heat sink temperatures. The design also adopts the use of refrigerants different from those used in similar applications. For $50 \mathrm{mK}$ operation, chrome potassium alum (CPA) provides better entropy capacity and lower temperature capability than the ferric ammonium alum used on Astro-E/E2 to reach $60 \mathrm{mK}$. For the warmer stages, research at GSFC over the last 10 years has identified a new material, gadolinium lithium fluoride (GLF) ${ }^{8}$ that has $30 \%$ higher entropy capacity per volume than gadolinium gallium garnet (GGG) ${ }^{9}$, yet lower temperature capability $(0.25 \mathrm{~K}$ versus $\sim 0.8 \mathrm{~K})$. This increases performance and opens up a much wider parameter space for optimizing the design, which is summarized in Table 2.

The components of each stage - salt pills, magnets, magnetic shields, Kevlar suspension and heat switches - also incorporate design and manufacturing processes recently developed for multi-stage ADRs ${ }^{10,11}$ as these aspects have been demonstrated to increase thermodynamic efficiency and reduce overall mass and size. A brief description of these components follows.

Table 2. ADR design summary.

\begin{tabular}{|c|c|c|c|}
\hline & $\underline{\text { Stage } 1}$ & $\underline{\text { Stage } 2}$ & $\underline{\text { Stage } 3}$ \\
\hline Refrigerant type & $\mathrm{CPA}$ & GLF & GLF \\
\hline Refrigerant mass & $270 \mathrm{~g}$ & $150 \mathrm{~g}$ & $150 \mathrm{~g}$ \\
\hline Magnetic field & $2 \mathrm{~T}$ & $3 \mathrm{~T}$ & $3 \mathrm{~T}$ \\
\hline Hold temperature, $T_{o p}$ & $0.05 \mathrm{~K}^{*}$ & $0.5 \mathrm{~K}$ & $1.2-4.5 \mathrm{~K}$ \\
\hline Demagnetization temperature & $0.8 \mathrm{~K}$ & $1.4 \mathrm{~K}$ & $4.5 \mathrm{~K}$ \\
\hline Cooling capacity at $\mathrm{T}_{\mathrm{op}}$ & $0.165 \mathrm{~J}$ & $\begin{array}{l}0.76 \mathrm{~J} @ 0.5 \mathrm{~K} \\
+2.91 \mathrm{~J} @ 0.8 \mathrm{~K}\end{array}$ & $\sim 2 \mathrm{~J}$ \\
\hline
\end{tabular}

\subsection{Salt Pills}

The $1^{\text {st }}$ stage stage refrigerant, CPA, is a hydrated salt that has a long history of use in ADRs. The challenge to its use is to provide a high conductance thermal bus and hermetic enclosure to ensure that heat can be efficiently transferred to/from the salt and that it remains hydrated. The design used is an adaptation of a concept developed for salt pills used in a continuous ADR which required unusually high thermal conductance. The thermal bus is cut from a single cylinder of electrolytic tough pitch (ETP) copper using wire electric discharge machining (EDM) to form a grid of copper wires connected at each end where a flange provides a bolting interface. The copper is welded inside a stainless steel tube, and the salt is grown from solution onto the copper wires. Approximately $10 \%$ of the resulting volume is copper, and we have achieved CPA fill fractions of $98-99 \%$.

The $2^{\text {nd }}$ and $3^{\text {rd }}$ stages use polycrystal GLF manufactured in $3 \mathrm{~mm}$ thick wafers. The proprietary manufacturing process by Konoshima Chemical Co., Japan achieves $99 \%$ of single-crystal density. The wafers are stacked inside a high-purity copper enclosure that is hermetically enclosed within a stainless steel sleeve and sealed with 1 atmosphere of ${ }^{3} \mathrm{He}$ for heat exchange. The two types of salt pill are shown in Figure 3. 

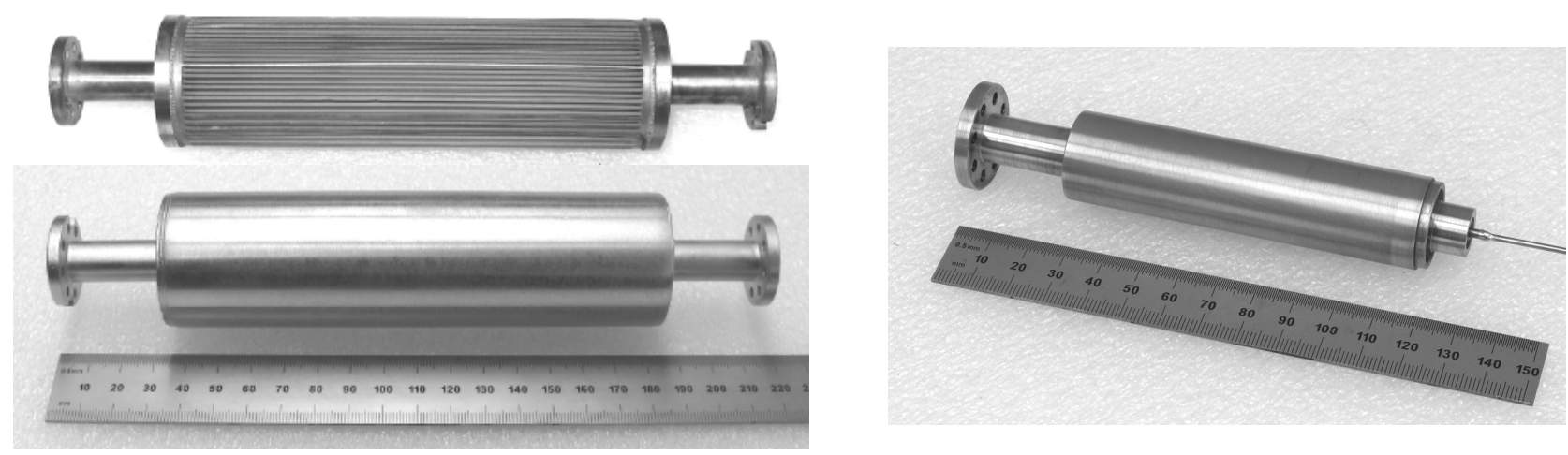

Figure 3. Partial and completed $1^{\text {st }}$ stage (at left) and $2^{\text {nd }} / 3^{\text {rd }}$ stage (at right) salt pill assemblies.

\subsection{Gas-Gap Heat Switches}

All 4 heat switches used in the ADR are active gas-gap switches. This technology has flight heritage ${ }^{4}$, and easily achieves the high thermal conductance between stages needed to meet the recycle time requirement. While we have developed passive gas-gap switches ${ }^{11}$ for use in multi-stage ADRs which could be used for 3 of the switches, the use of active switches gives more flexibility in operation and tolerance for off-nominal conditions, such as may be experienced with changes in performance of the JT coolers, failure of one of the shield coolers, or excessive parasitic heat loads.

The internal conductors of the switch are also cut from a single cylinder of ETP copper with wire EDM. The cutting is done in a single pass in a back and forth manner to yield interleaved copper fingers uniformly separated by $0.4 \mathrm{~mm}$. The two pieces are hermetically sealed in a containment tube (with end flanges), with a charcoal getter assembly sealed onto one end. The switch is filled with 1 atmosphere of ${ }^{3} \mathrm{He}$. The getter is heated with an external heater (a ruthenium oxide chip resistor) requiring only about $0.25 \mathrm{~mW}$ to reach $12 \mathrm{~K}$. The turn on/turnoff time is approximately 1 minute.

\subsection{Superconducting Magnets and Shields}

The superconducting magnets are manufactured - as are all other ADR components - in-house at GSFC. Although the diameter and length of the winding region is different for the 2T and 3T magnets, all consist of approximately 120,000 turns of $0.1 \mathrm{~mm}$ diameter (with Formvar coating) multi-filamentary NbTi wire ${ }^{12}$. The magnets are diode protected for quench survival, and have been shown to tolerate $125 \%$ of their nominal operating current of $2 \mathrm{~A}$, in vacuum, at ramp rates of up to $0.4 \mathrm{~A} / \mathrm{min}$.

The SXS detector array is not particularly susceptible to magnetic fields, but spacecraft components such as magnetometers in the attitude control system are, as are neighboring ADR stages. Two requirements for magnetic field attenuation have been levied on the ADR subsystem: fringing fields cannot exceed $10 \mathrm{mT}$ at the detector array location, and the magnets' dipole moment must be less than $10 \mathrm{~A} \cdot \mathrm{m}^{2}$ at full field. The latter is by far the more stringent requirement, and is achieved by enclosing each magnet in a vanadium permendur (also known as Hiperco 50) shield.

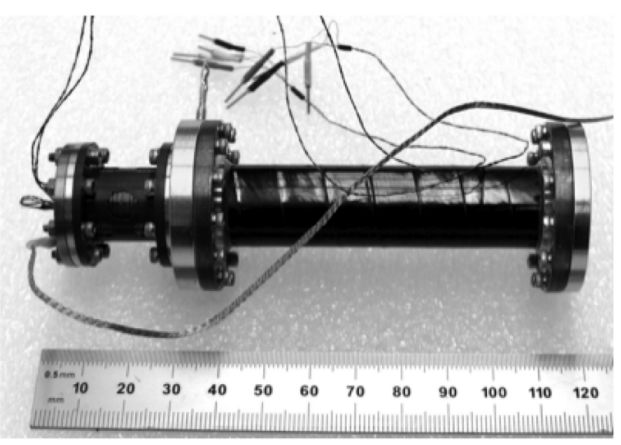

Figure 4. Active gas-gap heat switch with T300 carbon fiber composite containment shell. 

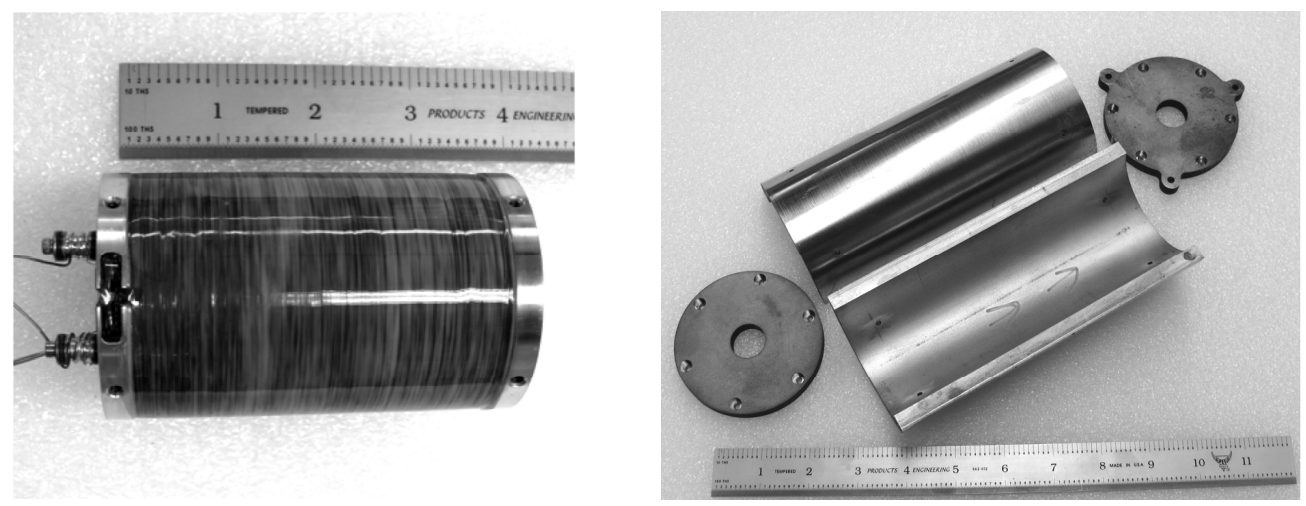

Figure 5. NbTi magnet and magnetic shield.

The shield is shaped to concentrate mass near the axial midpoint, where the maximum flux must be returned. Magnetic field modeling was performed using Magneto, and validated measurements using the simple cylindrical shields shown with the magnets in Figure 5. The optimized shields are being fabricated at present for the engineering model (EM) $\mathrm{ADR}$, and are predicted to yield a peak dipole moment of $1 \mathrm{~A} \cdot \mathrm{m}^{2}$ and peak field at the detectors of 0.1-0.2 $\mathrm{mT}$.

\subsection{Kevlar Suspension}

The salt pills in the 2-stage ADR where parasitic heat loads are most critical are suspended within their magnets by two separate Kevlar fiber suspension assemblies, shown in Figure 6. The combination provides kinematic control of motion in all 6 degrees of freedom. The forward "gimbal" assembly uses a spring loaded axial loop of Kevlar counterbalancing 3 loops angled at $45^{\circ}$ relative to the central axis. When bolted to the interface flange of each salt pill, it controls axial motion, and lateral motion of the forward end. The aft end assembly uses a central ring attached to the midpoints of each leg of a triangular loop of Kevlar that is spring loaded at all 3 vertices. The ring is split (not shown), allowing it to be clamped onto one end of each salt pill.

Each loop is made from 8 turns of a bundle of 200 Kevlar-29 fibers, except for the axial loop in the gimbal assembly which uses 16 turns. A single fisherman's knot is used to complete each loop. Braided rope with the same number of fibers is advertised ${ }^{13}$ to have a breaking strength of 65 pounds ( 290 Newtons), whereas the measured breaking strength of the loops, which should be twice this load, consistently falls between 90 and 95 pounds (400-423 Newtons). In their assemblies, the loops are spring loaded only to $\sim 25 \%$ of these values.

\section{ADR OPERATING MODES AND EXPECTED PERFORMANCE}

The ADR has two primary operating modes on orbit: 2-stage operation with liquid helium, and 3-stage operation without liquid helium.
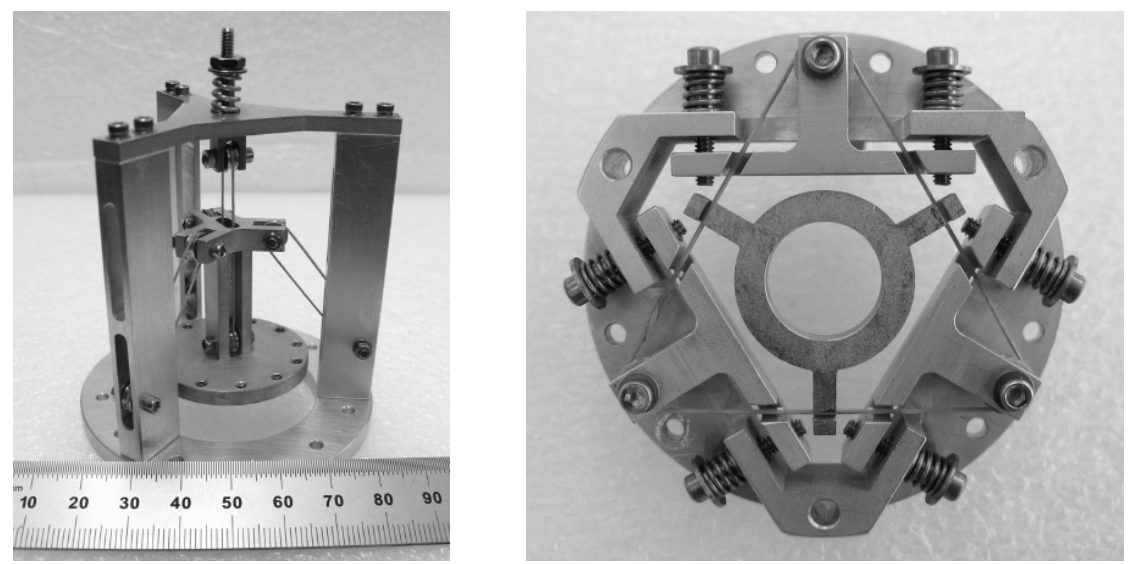

Figure 6. Forward "gimbal" (at left) and aft (at right) Kevlar suspension assemblies. 


\subsection{ADR Operation with Liquid Helium}

In designing the 2-stage portion of the CSI, a key consideration was the sequence used to recycle the ADR. There are several options: both stages can be magnetized simultaneously, or they can be recycled in sequence, either one being the first to be recycled. While simultaneous magnetization is generally faster, it results in larger magnetic fields and/or poorer low temperature performance. So the recycling is done in sequence, allowing the $1^{\text {st }}$ stage to be pre-cooled to a lower starting temperature for demagnetization and use a smaller magnet. The order in which the stages are recycled was determined during the optimization process in which the operating and demagnetization temperatures of the 2 stages were chosen. For the Astro-H design, the $2^{\text {nd }}$ stage will hold at $0.5 \mathrm{~K}$, but recycling of the $1^{\text {st }}$ stage (where it transfers heat to the $2^{\text {nd }}$ stage) will occur at $0.8 \mathrm{~K}$. In order to make the best use of the $2^{\text {nd }}$ stage's refrigerant, its cycle should end (i.e. the stage should run out of current) at the higher of the two temperatures. Thus the best sequence, at the end of a hold period, is to transfer heat from the $1^{\text {st }}$ stage to the $2^{\text {nd }}$ stage, then from the $2^{\text {nd }}$ stage to the bath, then cool both stages to their operating temperatures.

This process was modeled using a high-fidelity model in which the thermodynamic state of the ADR is calculated in real-time in response to outputs from the control system, and has subsequently been verified through testing of a breadboard ADR. The current and temperature profiles are shown in Figure 7 for the predicted heat loads given below:

Stage 1: $0.25 \mu \mathrm{W}$ detector load

$0.05 \mu \mathrm{W}$ through the Kevlar suspension to $1.3 \mathrm{~K}$

$\underline{0.09 \mu \mathrm{W}}$ through the heat switch to Stage 2 at $0.5 \mathrm{~K}$

$0.39 \mu \mathrm{W}$ total

Stage 2: $0.05 \mu \mathrm{W}$ through the Kevlar suspension to $1.3 \mathrm{~K}$

$\underline{0.85 \mu \mathrm{W}}$ through the heat switch to $1.3 \mathrm{~K}$

$0.90 \mu \mathrm{W}$ total

Given the cooling capacity listed in Table 2 and assuming a heat absorption efficiency of $85 \%$ (which has been verified by test), the hold time of the $1^{\text {st }}$ stage is predicted to be in excess of 100 hours.
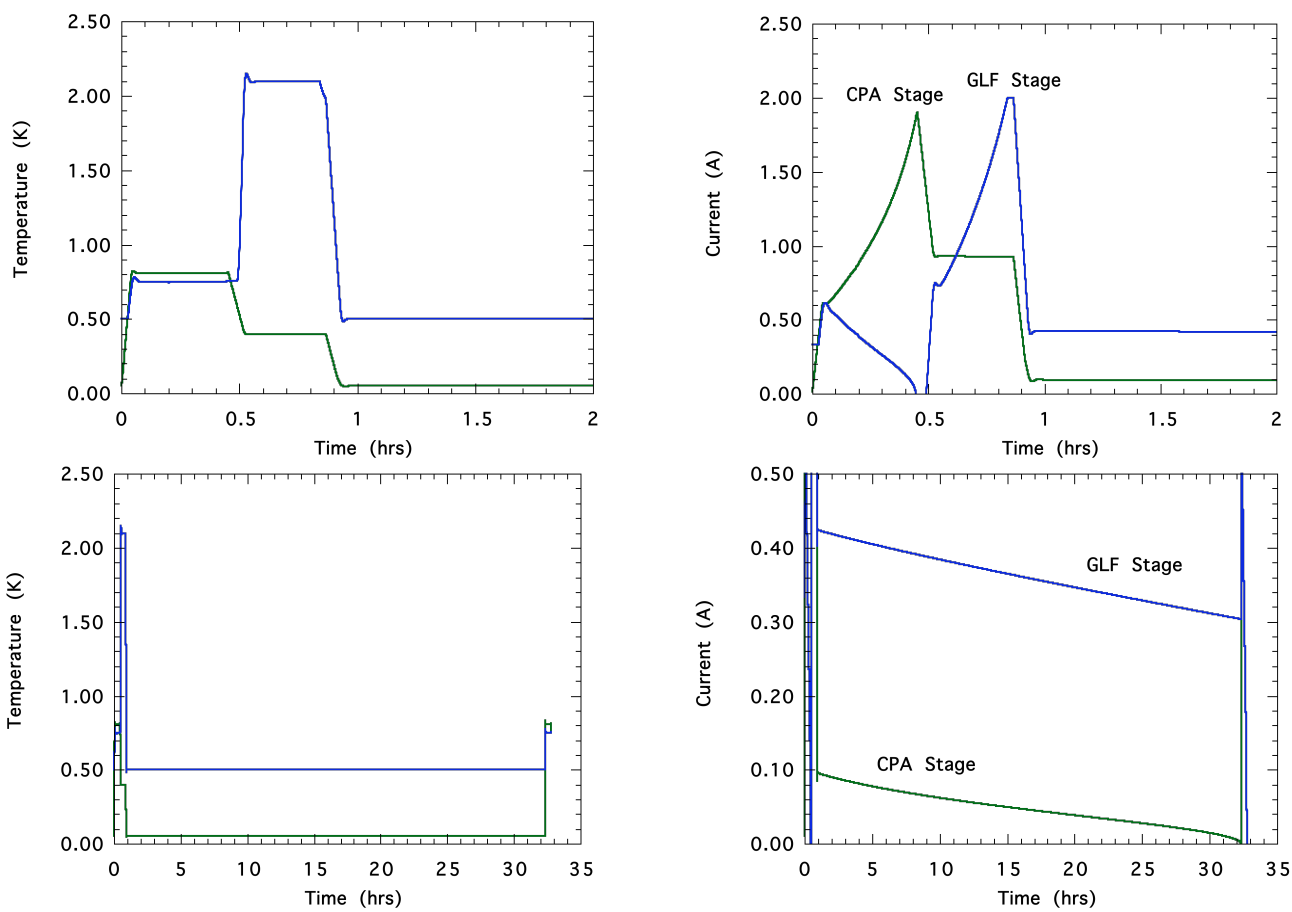

Figure 7. Temperature and current profiles for a complete cycle for operation with liquid helium. Upper graphs highlight the recycling period, and the lower graphs shows the extended hold time. For this simulation, heat loads on the ADR have been doubled to verify that the design carries at least $100 \%$ margin. 
Heat conducted along sensor wiring was assumed to be negligible, but this is currently being re-evaluated, as are other sources of heat such as radiation sneak paths, in light of recent measurements that show elevated parasitics that are not plausibly attributed to the heat switches and Kevlar suspension: $0.70 \mu \mathrm{W}$ to the $1^{\text {st }}$ stage, and $1.30 \mu \mathrm{W}$ to the $2^{\text {nd }}$ stage. Even at these values the ADR's hold time exceeds the 24 hour requirement, but it is expected that once the ADR is installed in a test dewar that is specially designed for testing the CSI, these excess loads will be eliminated.

The total heat output of the ADR during recycling, which has contributions from power used to activate the heat switches, hysteresis heat from the magnets and shields, and direct heat from the salt pills, is $10.0 \mathrm{~J}$. If this is produced every 100 hours (the predicted hold time), it represents a time-average load of only $27 \mu \mathrm{W}$. Even if the hold time is only 24 hours, the average load will only be $0.12 \mathrm{~mW}$, still well below the requirement.

\subsection{ADR Operation without Liquid Helium}

When the liquid helium is depleted, the operation of the ADR is changed significantly. The strategy is to use the $3^{\text {rd }}$ stage to maintain the helium tank at the lowest possible temperature by cycling back and forth in temperature to offload heat from the tank and transfer it to the JT, as shown in Figure 8. From the tank's perspective, this is a discrete process in which, for approximately half the 20 minute cycle (limited by the $20 \mathrm{~mW}$ available cooling power of the JT), the $3^{\text {rd }}$ stage is cold and absorbing heat from the tank to steadily cool it, and for the other half, the $3^{\text {rd }}$ stage is warm, rejecting heat to the JT, and the tank steadily warms under the heat loads from parasitic sources and the 2-stage ADR.

While this occurs, the $2^{\text {nd }}$ stage works only to stabilize the temperature of the detector housing. That is, its control system switches from regulation using a thermometer on the salt pill to one mounted on the base of the detector assembly. Effectively, the $2^{\text {nd }}$ and $3^{\text {rd }}$ stages form a 2-stage continuous ADR to provide a low temperature sink for the $1^{\text {st }}$ stage. In this mode, it is necessary for the heat switch that links the $2^{\text {nd }}$ stage to the helium bath to be on continuously. This was one motivation for re-designing the heat switch to require significantly less power than was needed by the Astro-E/E2 ADR's heat switch.

From thermal analysis performed by Sumitomo Heavy Industries, Japan, the contractor responsible for the design and production of the cryocoolers and helium dewar, the average heat load on the helium tank in this mode is approximately $0.76 \mathrm{~mW}$, to which is added approximately $0.30 \mathrm{~mW}$ steady heat load from the 2 ADR stages thermally connected to the CSI plate. The cooling capacity of the $3^{\text {rd }}$ stage at, for example, $1.2 \mathrm{~K}$, using $3 \mathrm{~T}$ and demagnetizing from $4.6 \mathrm{~K}$, is $3.1 \mathrm{~J}$. Cycle periods of 20 minutes or less are possible, yielding a gross cooling power of over $2.5 \mathrm{~mW}$. That the cooling power is in excess of the steady heat load on the helium tank allows the $2^{\text {nd }}$ stage to store up a cooling reserve. This reserve is used to recycle the $1^{\text {st }}$ stage on a daily basis.

The amount of excess cooling the $3^{\text {rd }}$ stage produces is a function of the helium tank temperature, so in operation a balance will have to be struck between trying to cool the tank as low as possible - to minimize parasitic loads on the detector and ADR - and accumulating cooling capacity at the rate needed to periodically recycle the $1^{\text {st }}$ stage. This is expected to be near $1.2 \mathrm{~K}$.

Modeling of this modified cycle has been performed, along with transient thermal analysis of gradients within the CSI plate during the ADR's operation. Both confirm that it should be possible to stabilize the detector housing temperature to within $0.5 \mathrm{mK}$ at all times, well within the requirement of $1 \mathrm{mK}$ over 10 minute periods.
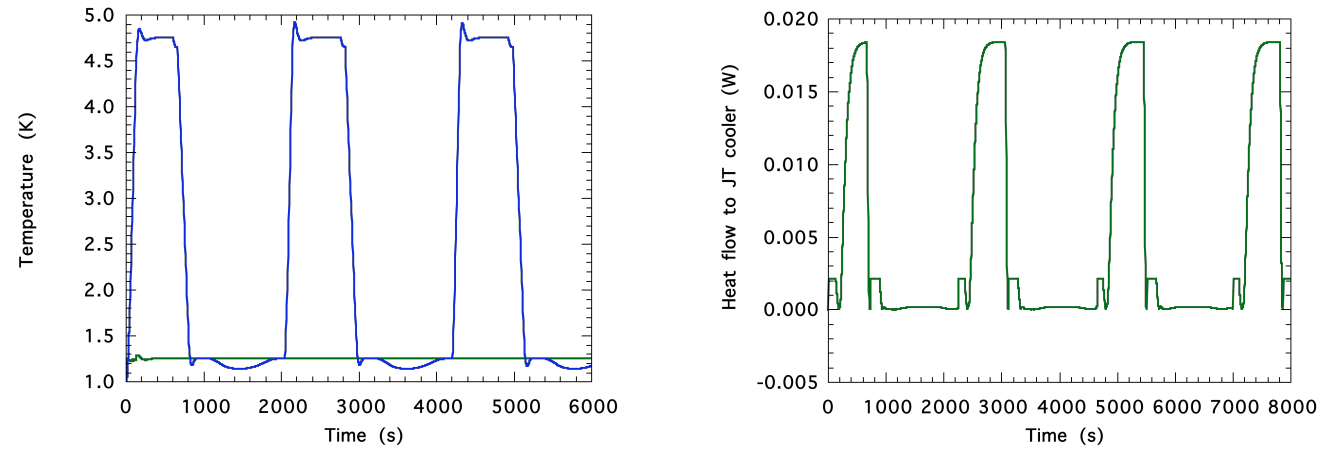

Figure 8. Temperature and heat load profiles for the $3^{\text {rd }}$ stage as it cycles to cool the helium tank. In the left graph, the temperature of the second stage is the flat line at $1.25 \mathrm{~K}$. 
Because the $1^{\text {st }}$ stage's heat switch now has a thermal connection to $\sim 1.2 \mathrm{~K}$ during its hold time, rather than $0.5 \mathrm{~K}$ as it does when operating with liquid helium, the hold time is significantly shorter. The parasitic load from the heat switch will be $0.78 \mu \mathrm{W}$, giving a total of $1.08 \mu \mathrm{W}$. Additionally, the simplest operation of the $1^{\text {st }}$ stage would have it recycle directly to the $2^{\text {nd }}$ stage/helium tank at $1.2 \mathrm{~K}$, rather than $0.8 \mathrm{~K}$. The reduction in cooling capacity at $50 \mathrm{mK}$ is about $30 \%$, yielding an estimated hold time of 23 hours.

Since this is not considered to be a failure mode, and full operational redundancy is required, it is important to have appreciable margin on performance at this stage of the project. One way to improve the situation is to thermally decouple the $2^{\text {nd }}$ stage from the helium tank and demagnetize to $\sim 0.8 \mathrm{~K}$ when the $1^{\text {st }}$ stage is recycled. Afterward, it can warm back to $1.2 \mathrm{~K}$ and resume regulating the helium tank temperature. This will restore the $1^{\text {st }}$ stage to its nominal cooling capacity at $50 \mathrm{mK}$, and disrupt the detector housing temperature only at a time when stability is not required. The hold time of the $1^{\text {st }}$ stage in this scenario is 33 hours.

\section{SUMMARY}

The redundant, hybrid cryogenic system which forms the basis of the SXS instrument on Astro-H poses significant challenges to the design of the ADR. Moreover, the configuration of the ADR is constrained by several factors: 1) available space within the helium cryostat, 2) the need to operate with full redundancy not only with two heat sinks at two very different temperatures $(1.3 \mathrm{~K}$ and $4.5 \mathrm{~K})$, but even if one of those systems should fail, 3 ) the need to minimize parasitic, hysteresis, and direct heat loads to the helium tank, particularly during cryogen-free operation, and 4) the need to operate the $3 \mathrm{rd}$ stage within the available cooling power of the ${ }^{4} \mathrm{He} \mathrm{JT}$ cooler.

So far as it has been possible to model and directly verify through measurements with a breadboard ADR, the 3-stage design presented meets all of the performance requirements for the SXS instrument. In fact, for operation with liquid helium, demonstrated performance of the 2-stage breadboard shows $100 \%$ margin on hold time, and for the lower, predicted heat loads, 300\% margin. Fabrication of the EM ADR is currently underway, expected to be completed in late August, 2010.

\section{REFERENCES}

[1] Mitsuda K, et al., "The X-Ray Microcalorimeter on the NeXT Mission”, Proc. SPIE vol. 7011, 701102K-1 (2008). [2] Fujimoto R, et al., "Cooling system for the Soft X-ray Spectrometer (SXS) onboard ASTRO-H”, Proc. SPIE vol. 7732 (2010).

[3] Serlemitsos AT, SanSebastian M and Kunes E, "Design of a spaceworthy adiabatic demagnetization refrigerator," Cryogenics 32, 117-124 (1992).

[4] Kelley RL, et al., “The Suzaku High Resolution X-Ray Spectrometer,” Publ. Astron. Soc. Japan 59, S77-112 (2007).

[5] Porter FS, Almy R, Apodaca E, Figueroa-Feliciano E, Galeazzi M, Kelley RL, McCammon D, Stahle CK,

Szymkowiak AE, Sanders WT, "Observations of the soft X-ray background with the XQC microcalorimeter sounding rocket”, Nucl. Instr. and Meth, 444, 175 (2000).

[6] Tuttle JG, Shirron PJ, DiPirro MJ, Jackson M, Behr J, Kunes E, and Hait T, "The HAWC and SAFIRE Adiabatic Demagnetization Refrigerators", Cryogenics 41, 781-787 (2002).

[7] Voellmer GM, Jackson ML, Shirron PJ, and Tuttle JG, “A Cryogenic, Insulating Suspension System for the HAWC and SAFIRE ADRs”, Proc. SPIE Vol. 4850, 1070 (2003).

[8] Numazawa T, Kamiya K, Shirron PJ, DiPirro MJ, Matsumoto K, "Magnetocaloric Effect of Polycrystal GdLiF4 for Adiabatic Demagnetization Refrigeration", AIP Conference Proceedings 850, 1579-1580 (2006).

[9] E.W. Hornung, R.A. Fisher, G.E. Brodale and W.F. Giauque, "Magnetothermodynamics of gadolinium gallium garnet", J. Chem. Phys. 61, 282 (1974).

[10] Shirron, P.J., Canavan, E.R., DiPirro, M.J., Tuttle, J.G., and Yeager, C.J., “A Multi-Stage Continuous Duty Adiabatic Demagnetization Refrigerator," in Adv. Cryo. Eng. 45B, 1629-1638 (2000).

[11] Shirron PJ, Canavan ER, DiPirro MJ, Jackson M, Panek JS, Tuttle JG. "Passive Gas-Gap Heat Switches for Use in Adiabatic Demagnetization Refrigerators”, Adv. Cryo. Eng. 47, 1175-1182 (2002).

[12] Multi-filamentary NbTi wire (15 filaments, 1.4:1 Cu:NbTi ratio) from Supercon, Inc., 830 Boston Turnpike, Shrewsbury, MA 01545.

[13] Kevlar 29, E. I. DuPont de Nemours, http://www.dupont.com/Kevlar/en_US/index.html 